\title{
Hypoxic-ischaemic encephalopathy after near miss sudden infant death syndrome
}

\author{
J E C CONSTANTINOU, J GILLIS, R A OUVRIER, AND P M RAHILLY
}

Departments of Neurology and Intensive Care, Royal Alexandra Hospital for Children, Sydney, Australia

SUMmARY Between 1982 and 1985, 14 infants aged 3-26 weeks presented with severe hypoxic episodes as a result of the 'near miss' sudden infant death syndrome (SIDS). They all had metabolic acidosis, cardiovascular instability, acute renal failure, ischaemic colitis, or acute neurological dysfunction. Investigation of the cause excluded infection and trauma, or a primary metabolic, pulmonary, cardiac, or seizure disorder. Seven infants were deeply comatose on admission, never regained consciousness, and died within 60 hours. A characteristic evolution of hypoxic-ischaemic encephalopathy not previously clearly described after near miss SIDS was seen in the seven who lived. Five of the seven were conscious within one hour of resuscitation and showed a striking interval of near normality before neurological deterioration that was characterised by status epilepticus, deep coma, and brain stem dysfunction from 36-96 hours after the event. A biphasic course was not apparent in the remaining two, each of whom was comatose on admission, though refractory seizures did develop. Computed tomograms of the brain more than a week after the event showed cortical infarction or cerebral atrophy. Six of the survivors, followed up from 16-55 months, have serious residual deficits including spastic quadriplegia, delayed development, cortical blindness, or infantile spasms.

'Near miss' sudden infant death syndrome (SIDS) refers to the recognised clinical phenomenon of the rescue of an apparently healthy infant from a life threatening event. The infant is found limp, apparently unconscious, not breathing, pale or cyanotic, and requires stimulation or more vigorous resuscitation. Once specific toxic, metabolic, infectious, traumatic, and other identifiable causes for this life threatening event have been excluded, there remains a set of infants who for unknown reasons have experienced an inexplicable episode of near death. ${ }^{1-3}$

The vast number of publications on near miss SIDS suggests that infants either die or recover rapidly following such an event. ${ }^{45}$ At most, subtle neurological abnormalities may occur. ${ }^{6} 7$ Only a single report suggests that significant neurological dysfunction may occur. ${ }^{8}$

In order to clarify whether near miss SIDS is associated with considerable morbidity, a retrospective review was conducted of infants with a history suggestive of near miss SIDS and clinical evidence of hypoxic derangement of a number of organ systems, including the central nervous system, admitted to the intensive care unit of the Royal Alexandra Hospital for Children, Sydney.

\section{Patients and methods}

The case histories of all infants admitted to the intensive care unit of The Royal Alexandra Hospital for Children, Sydney, for the period 1 June 1982 to 30 September 1985 were reviewed to identify those infants who had sustained a serious hypoxic injury as a result of near miss SIDS.

All subjects fulfilled the accepted criteria for the diagnosis of near miss SIDS. They had been found pale, cyanosed, limp or not breathing, and had required vigorous stimulation or cardiopulmonary resuscitation because death was thought to be imminent. In all infants, there was a lack of pathological events during pregnancy and delivery, including prematurity, and their neurological state seemed normal before the life threatening event. There was neither a history of seizures nor of fever.

Evidence of a hypoxic insult during admission consisted of metabolic acidosis (arterial pH $7 \cdot 0$ or less on presentation) or of cardiovascular instability (hypotension - that is, systolic blood pressure less than $40 \mathrm{~mm} \mathrm{Hg}$ associated with poor peripheral perfusion), acute renal failure (absent or urinary output decreased to less than $1 \mathrm{ml} / \mathrm{kg} /$ hour, or serum creatinine concentration of over $100 \mu \mathrm{mol} / \mathrm{l})$, 
ischaemic colitis (profuse watery, bloodstained diarrhoea with no other bacteriological, toxic, or surgical cause), or acute neurological dysfunction (deep coma or convulsions). Deep coma was diagnosed when the infant did not open his eyes (either spontaneously or in response to noise), made no sound, and made no purposeful response to localise or resist painful stimuli.

Infants were included in the study only if no obvious cause (sepsis, trauma, metabolic disorder, or primary pulmonary, abdominal, or cardiac abnormality) had been found for the life threatening event after extensive investigation during the hospital admission. Apart from detailed physical examination, this investigation included full blood count, estimations of the serum concentrations of electrolytes, ammonia, glucose, calcium, and magnesium, liver function tests, urinary amino acid screen, and organic acid profile by gas liquid chromatography. Cerebrospinal fluid was analysed, and viral and bacteriological cultures of blood, urine, stool, and cerebrospinal fluid were made. Chest radiography, electrocardiograms, and skeletal surveys were also carried out. Necropsies on those who died failed to establish a cause of death other than the primary hypoxic event.

The hospital records of those who lived were reviewed with particular reference to neurological state in the acute period. Serial electroencephalographic traces and cerebral computed tomograms were also studied. The neurodevelopmental outcome in the long term survivors, followed up clinically by the attending paediatrician, was recorded.

\section{Results}

Fourteen infants from 3-26 weeks of age (mean 13 weeks) satisfied criteria for the diagnosis of a near miss SIDS event with a significant hypoxic insult. There were seven boys and seven girls. One child (case 1) has been previously reported and was found to have an incidental stage I neural crest tumour that resolved spontaneously without treatment. Urinary catecholamine excretion was normal. ${ }^{9}$ As a comparison over the same period of time 35 other infants were admitted to the general hospital wards having had a near miss SIDS event that was not associated with hypoxic sequelae.

All infants were seen in hospital within 90 minutes of the life threatening event; six of the infants died within 24 hours. A further infant was withdrawn from life support systems after 60 hours because of brain death. Seven lived. All the children had previously been well and had not given cause for medical concern before the near miss SIDS event. Of those who lived, two had been slightly irritable and two had rhinorrhoea for a day or two before admission. Of those who died, two had been slightly irritable and one had had rhinorrhoea.

Of the seven who died, six were judged to have had cardiorespiratory arrest either at home by paramedical personnel, or in a hospital emergency department. Resuscitation consisted of intubation, artificial ventilation, and adrenaline given either intravenously or through the endotracheal tube. Estimated duration of arrest before resumption of cardiac output varied from 10-55 minutes. One other infant who died (case 13) presented with agonal gasping and unrecordable blood pressure. All these infants remained hypotensive and required volume expansion and inotropic support. Four developed signs of acute renal failure, and four ischaemic colitis.

After resuscitation at home all seven who lived had established spontaneous respirations by the time that they arrived in the hospital emergency department. Resuscitation had consisted of vigorous stimulation, mouth to mouth resuscitation or cardiac massage. One infant required resuscitation with a bag and mask (case 7). Three of these infants required intravenous colloid and inotropic support to maintain adequate cardiac output. Three developed ischaemic colitis, and two acute renal failure (one of whom required peritoneal dialysis).

Table 1 shows the evidence of hypoxic derangement during admission in the two groups. Metabolic acidosis on presentation, continued cardiovascular instability, acute renal failure, or ischaemic colitis occurred more often among those who died. In two infants (cases 2 and 4) acute neurological dysfunction was the only evidence of an hypoxic injury. Liver function tests showed a mild increase in the transaminase activity in two cases (cases 1 and 6). Three infants (cases 1, 3, and 4) developed hyponatraemia secondary to inappropriate antidiuretic hormone secretion during admission.

ACUTE NEUROLOGICAL COURSE

All seven who died were in deep coma at their initial presentation and remained so until the time of death. In all but one the pupils did not react to light at initial presentation. Three patients (cases 8, 10, and 13) developed seizures before death.

Two distinct patterns of neurological dysfunction were seen in the seven survivors. All had normal pupillary reactions at the initial assessment; details of their neurological course and outcome are shown in table 2.

Five of the infants (cases 1-5) showed a biphasic evolution of neurologic dysfunction characterised by 
Table 1 Clinical signs of hypoxic insult

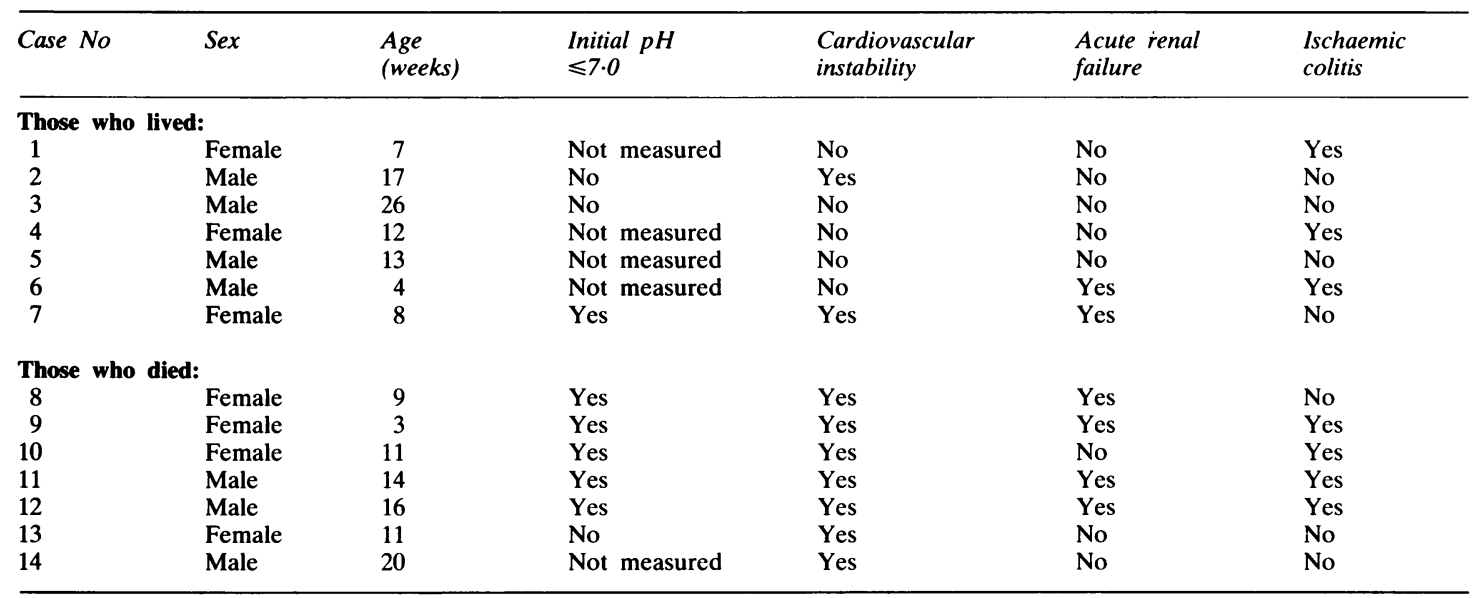

Table 2 Details of neurological course and outcome of those who lived

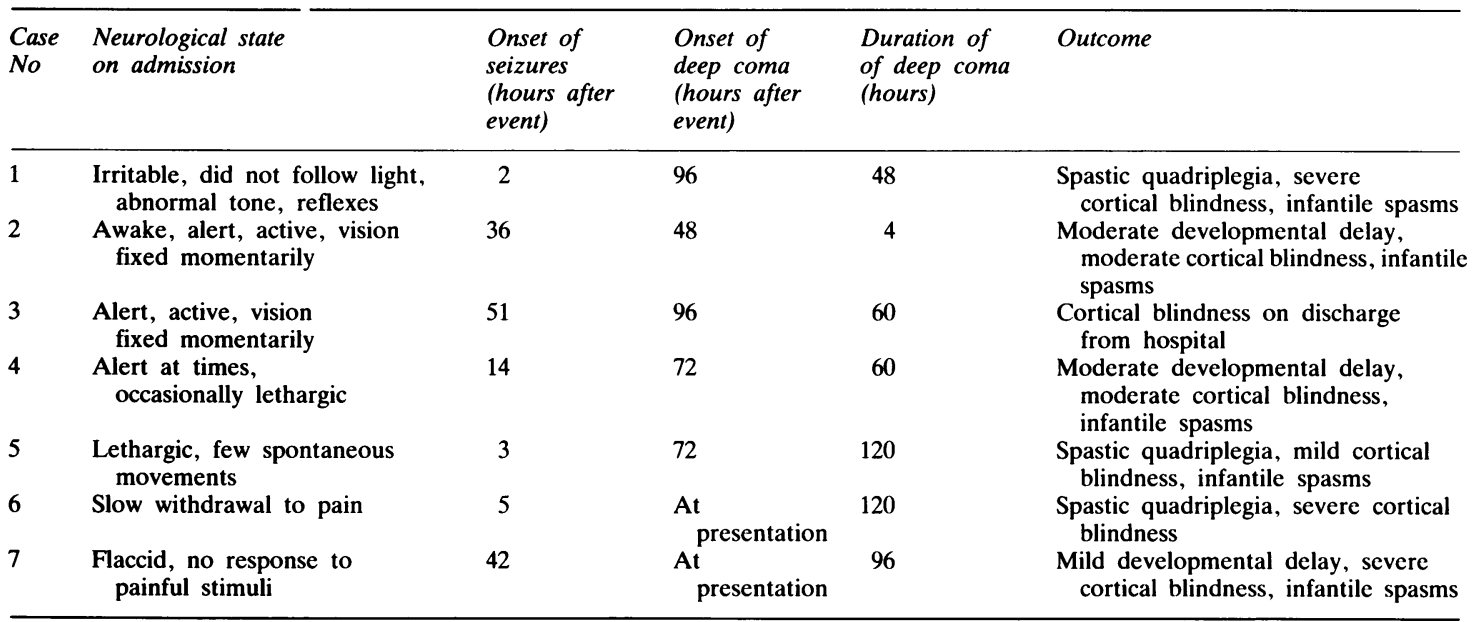

an initial period of near normality and subsequent neurological deterioration. These five infants were conscious and rouseable within one hour of resuscitation. They were awake, alert and active, or irritable or lethargic with only minor abnormalities of tone and deep tendon reflexes. In three cases there were concerns about their ability to fix their gaze. This striking interval of near normality continued until the onset of seizures from 2-51 hours (mean 21 hours) after the event. In one patient (case 1) isolated myoclonic jerks, evident at initial presentation, did not recur until 18 hours after the event. The seizures (initially tonic or multifocal and clonic) increased in frequency and duration with eventual secondary generalisation from 28-72 hours (mean 51 hours) after the event. Status epilepticus, defined as a generalised convulsion which lasted more than 30 minutes or as serial convulsions between which there was no return of consciousness, occurred in all five. ${ }^{10}$ Two patients in this group required an infusion of thiopentone to control the seizures. Associated with increasing severity of the convulsions there was overall deterioration in the infants' neurological condition, with progression to deep coma from 48-96 hours after the event. Deep coma, as defined previously, persisted from 4-120 hours (mean 58 hours). Signs of brain stem dysfunction-that is, no oculocephalic responses 
(cases 3 and 4), pupillary inequality (case 1), and divergent eyes (case 5)-occurred in four of the patients in association with deep coma. The fontanelle was bulging in four.

The other two survivors (cases 6 and 7) were deeply comatose on admission. A biphasic course was not apparent though refractory seizures did develop. One of these patients (case 7) also required an infusion of thiopentone.

Cranial computed tomograms were obtained in all except one of the patients (case 6). Early scans (obtained within eight days of the life threatening event) were normal (cases 2 and 4), or showed focal (case 3), or generalised (case 1) hypodensity, suggestive of cerebral oedema. Cranial ultrasound in this last case showed increased density in the subarachnoid space suggestive of blood, but this was not confirmed on computed tomography. Cranial ultrasound in case 6 showed no abnormality. Computed tomograms obtained after the first eight days were normal in one case (case 2), or showed changes of cerebral atrophy in one patient (case 3), and frontal, temporal, or occipital low attenuation areas consistent with watershed infarction in three (cases 4,5 , and 7).

Ictal activity on an abnormal background rhythm of low amplitude slow waves was seen in five of the six patients in whom electroencephalographic traces were obtained within one week of the life threatening event. This activity consisted of either rhythmic spike and slow wave resembling paroxysmal lateralising epileptiform discharges in three cases (cases 2,6 , and 7), or rhythmic spikes in two (cases 4 and 5). The discharges characteristically arose unilaterally in alternate hemispheres, or occasionally synchronously in both. The one patient in whom ictal activity was not seen (case 3 ) was receiving an infusion of thiopentone at the time of the record. Electroencephalographic records after the first week showed persistence of the abnormal background rhythm with the disappearance of ictal activity.

\section{OUTCOME}

Six of the seven infants (cases $1,2,4,5,6$, and 7) have been followed up for periods of one year four months to four years seven months (mean two years eight months) and they all show evidence of serious handicap. One child (case 3) was unfortunately lost to follow up, but was said to be developmentally normal one year after the event when reviewed by his local doctor.

Three of the survivors (cases 1, 5, and 6) are severely handicapped by spastic quadriplegia and live in institutions. The remaining three (cases 2,4 , and 7) are mildly or moderately developmentally delayed as assessed with the Reynell-Zinkin De- velopmental scales for young visually handicapped children and the Maxfield-Bucholz scale of social maturity.

All survivors are cortically blind. Three of them (cases 1, 6, and 7) are severely impaired and have light perception only. One (case 5) can distinguish large objects at one metre, and two (cases 2 and 4) can do so at three metres. Visual function in these two is characterised by visual inattention and variability. Case 3 was cortically blind at discharge from hospital. He was said to have normal vision one year after the event.

Five infants developed infantile spasms with electroencephalographic changes of true or modified hypsarrhythmia.

\section{Discussion}

The exact association between near miss SIDS and SIDS is unclear. Most series emphasise a possible overlap between the two (similar age distribution and more male and preterm infants and siblings of SIDS victims than in the general population). Babies referred as 'near miss' infants probably are, as a group, at increased risk for sudden death. ${ }^{1-4} 11-13$

Near miss SIDS may represent a pathophysiological disorder characterised by prolonged central or mixed apnoea, the end result of which may be sudden death. ${ }^{1314}$ It has been suggested that a child either dies from SIDS, or recovers from near miss SIDS after a life threatening apnoea, and that this recovery is complete. ${ }^{45}$ The literature does not address the possibility that recovery from near miss SIDS is not always an 'all or nothing' event. Significant hypoxic sequelae are not reported.

This study identifies a selected group of infants who were resuscitated from a 'near miss' event and who also showed clinical evidence of hypoxic derangement of at least one, and often many, organ systems. Necropsies showed findings consistent with a diagnosis of SIDS. Careful clinical and laboratory investigations in the surviving infants excluded specific toxic, metabolic, infective, and other causes. All the infants were neurologically normal before the event. The infants did not fulfil the criteria for the diagnosis of the syndrome of haemorrhagic shock and encephalopathy which, in addition to encephalopathy and convulsions, is also characterised by a high fever, severe hepatic dysfunction, and a haemorrhagic diathesis. 1516

Mortality in this selected group was high and the survivors developed serious neurological complications. In most of the survivors the acute neurological course started with a normal period, and coma and convulsions developed later. The long term outlook 
was poor, with cortical blindness, infantile spasms, and varying degrees of developmental delay.

The findings presented here contrast with those that suggest that infants either die or recover rapidly after a 'near miss' event. By selecting a group with hypoxic derangement, many infants who do recover rapidly have been excluded. It remains surprising, however, that reports of significant neurological deficits after near miss SIDS are infrequent. Aubourg et al described 12 infants with status epilepticus in eight of whom a history of an unrecognised life threatening event 36 hours to four days before the onset of epilepsy, was retrospectively obtained. $^{8}$ These infants shared many features with the babies reported here including an interval of near normality, similar electroencephalographic features, and a high frequency of handicap including cortical blindness, a well known manifestation of hypoxic insult. ${ }^{17}$

Interestingly, Kelly et al described a number of infants who required bag and mask resuscitation from a near miss episode. These infants developed transient lethargy, poor fixation on objects, hyperreflexia, and clonus. ${ }^{4}$ In addition the same group reported that some infants who had repeated, severe near miss episodes requiring resuscitation or vigorous stimulation later developed a seizure disorder. ${ }^{11}$

The poor prognosis of this selected group of infants is not surprising. Deep coma for even a few hours may be associated with poor neurological recovery in older children. ${ }^{18}$ Fixed, dilated pupils, the continued need for cardiopulmonary resuscitation, and severe metabolic derangement at initial assessment all predict a poor prognosis in studies of near drowning. ${ }^{19}{ }^{20}$ Snyder et al, however, reported that normal consciousness after an anoxaemic episode and before the onset of seizures is associated with a comparatively favourable outcome. ${ }^{21}{ }^{22}$ It is apparent that in the babies described here the period of near normality after the hypoxic-ischaemic insult was not associated with a good prognosis.

As hypoxic-ischaemic encephalopathy after near miss SIDS has not previously been clearly delineated, the most interesting aspect of this study is the clearly biphasic course of five of the survivors. The interval of near normality before the secondary neurological deterioration is similar in many ways to the early period of hyperalertness that typically gives way to increasing stupor and seizures in severe perinatal hypoxic-ischaemic encephalopathy. ${ }^{23}$

The phenomenon of delayed neurological deterioration after anoxia, first described by Plum and Posner, should be clearly distinguished from the delayed onset of seizures and coma in the infants described in this report. ${ }^{24} 25$ The former condition predominantly affects white matter, is thought to be due to demyelination, usually occurs from two to eight days after the anoxic event, and is characterised by pyramidal and extrapyramidal signs and symptoms without seizures. The condition reported in this paper probably reflects acute hypoxicischaemic encephalopathy, a triad of multifocal necrosis, vasogenic oedema, and raised intracranial pressure affecting primarily the grey matter. ${ }^{26}$

The results of recent experimental work have suggested that the clinical characteristics of acute hypoxaemic encephalopathy-that is, the hyperalert state and the seizure activity, may relate to hyperactivity of excitatory synapses that are highly concentrated in those regions of the developing brain vulnerable to hypoxic-ischaemic damage. Excessive release of neurotransmitters (particularly glutamate) triggers a cascade of biochemical reactions and potentially lethal ionic shifts with eventual neuronal death taking place over hours or days. ${ }^{27}$

Specific treatment, including glutamate antagonists, may soon become available so that this cascade of biochemical events can be interrupted. Flunarizine hydrochloride, a commercially available calcium channel blocker, has been shown to block the effects of brain perinatal hypoxaemia-ischaemia in an in vivo model and offers exciting prospects for the reduction of the serious consequences of hypoxaemic-ischaemic encephalopathy. ${ }^{28}$ It is possible that the early use of anticonvulsants may prevent refractory seizures that coincide with deteriorations in mental state.

Before such treatments are available, knowledge of the present poor prognosis of this unfortunate group of infants may be of value as a guide to acute treatment regimens, in parental counselling, and in the anticipation of long term rehabilitation needs.

We thank Judith McDonald for preparing the manuscript.

\section{References}

${ }^{1}$ Read DJC, Jeffery HE, Rahilly P. Sudden infant death syndrome and suspected "near-miss": an overview for clinicians. Med J Aust 1982;1:82-7.

2 Valdes-Dapena MA. Sudden infant death syndrome: a review of the medical literature 1974-1979. Pediatrics 1980;66:597-614.

${ }^{3}$ Dunne K, Matthews T. Near-miss sudden infant death syndrome: clinical findings and management. Pediatrics 1987;79: 889-93.

${ }^{4}$ Kelly DH, Shannon SC, O'Connell K. Care of infants with "near-miss" sudden infant death syndrome. Pediatrics 1978;61: $511-4$.

5 Deonna T, Micheli JL. Infantile status epilepticus as a complication of "near-miss" sudden infant death. Dev Med Child Neurol 1986;28:120-1.

${ }^{6}$ Korobkin R, Guilleminault C. Neurological abnormalities in near miss for sudden infant death syndrome infants. Pediatrics 1979;64:369-74.

7 Deykin E, Bauman ML, Kelly DH, et al. Apnea of infancy and subsequent neurologic, cognitive and behavioral status. Pediatrics 1984;73:638-45. 


\section{Constantinou, Gillis, Ouvrier, and Rahilly}

${ }^{8}$ Aubourg P, Dulac O, Plouin P, et al. Infantile status epilepticus as a complication of near-miss sudden infant death. Dev Med Child Neurol 1985;27:40-8.

9 Howman-Giles R, Rahilly PM. Technetium-99m methylene diphosphonate accumulation in the diaphragm after severe ischemia. Clin Nucl Med 1983;8:416-7.

${ }^{10}$ Haslam R. Status epilepticus in children. In: Ouvrier RA, ed. Controversies in childhood epilepsy. Amsterdam: Excerpta Medica, 1986:38-46.

1 Oren J, Kelly D, Shannon DC. Identification of a high-risk group of sudden infant death syndrome among infants who were resuscitated for sleep apnea. Pediatrics 1986;77:495-9.

12 Ariagno LA, Guilleminault C, Korokbin R, et al. "Near-miss" for sudden infant death syndrome infants: a clinical problem. Pediatrics 1983;71:726-30.

13 Steinschneider A. Prolonged apnea and the sudden infant death syndrome: clinical and laboratory observations. Pediatrics 1972; 50:646-54.

14 Baba N, Quattrochi JJ, Reiner CB, et al. Possible role of the brain stem in sudden infant death syndrome. JAMA 1983;249: 2789-91.

15 Levin M, Hjelm M, Kay JD, et al. Haemorrhagic shock and encephalopathy: a new syndrome with a high mortality in young children. Lancet 1983;ii:64-7.

16 Whittington LK, Roscelli JD, Parry WH. Hemorrhagic shock and encephalopathy: further description of a new syndrome. J Pediatr 1985;106:599-602.

17 Whiting S, Jan JE, Wong PKH, et al. Permanent cortical visual impairment in children. Dev Med Child Neurol 1985;27:730-9.

${ }^{18}$ Margolis LH, Shaywitz BA. The outcome of prolonged coma in childhood. Pediatrics 1980;65:477-83.
${ }^{19}$ Frates RC. Analysis of predictive factors in the assessment of warm-water near drowning in children. Am J Dis Child 1981;135:1006-8.

${ }^{20}$ Kruus S, Bergstrom L, Suutarinen T, et al. The prognosis of near-drowned children. Acta Paediatr Scand 1979;68:315-22.

${ }^{21}$ Snyder BD, Loewenson RB, Gumnit RJ, et al. Neurologic prognosis after cardiopulmonary arrest. II: Level of consciousness. Neurology 1980;30:52-8.

${ }^{22}$ Snyder BD, Hauser WA, Loewenson RB, et al. Neurologic prognosis after cardiopulmonary arrest. III: Seizure activity. Neurology 1980;30:1292-7.

23 Sarnat HB, Sarnat MS. Neonatal encephalopathy following fetal distress. Arch Neurol 1976;33:696-705.

24 Plum F, Posner JB, Hain RF. Delayed neurological deterioration after anoxia. Arch Intern Med 1962;110:18-25.

25 Antony JH. Relapsing encephalopathy after hypoxia. J Pediatr 1978;92:433-4.

${ }^{26}$ Brann AW. Hypoxic ischaemic encephalopathy (asphyxia). Pediatr Clin North Am 1986;33:451-64.

27 Johnston MV, Silverstein FS. New insights into mechanisms of neuronal damage in the developing brain. Pediatr Neurosci 1985-86;12:87-9.

${ }^{28}$ Silverstein F, Buchanan K, Hudson C, et al. Flunarazine limits hypoxia-ischemia induced morphologic injury in immature rat brain. Stroke 1986;17:477-82.

Correspondence to Dr J Gillis, Department of Intensive Care, Royal Alexandra Hospital for Children, PO Box 34, Camperdown, NSW 2050, Australia.

Accepted 29 November 1988 